\title{
Attentional focusing with quantifiers in production and comprehension
}

\author{
ANTHONY J. SANFORD, LINDA M. MOXEY, and KEVIN B. PATERSON \\ University of Glasgow, Glasgow, Scotland
}

\begin{abstract}
There is a very large number of quantifiers in English, so many that it seems impossible that the only information that they convey is about amounts. Building on the earlier work of Moxey and Sanford (1987), we report three experiments showing that positive and negative quantifiers focus on different subsets of the logical possibilities that quantifiers allow semantically. Experiments 1 and 2 feature a continuation task with quantifiers that span a full range of denotations (from near $0 \%$ to near $100 \%$ ) and show that the effect is not restricted to quantifiers denoting small amounts. This enables a distinction to be made between generalization and complement set focus proper. The focus effects extend to comprehension, as shown by a self-paced reading study (Experiment 3). It is noted that the focus effects obtained are compatible with findings from earlier work by Just and Carpenter (1971), which used a verification paradigm, and in fact these effects constitute a direct test of inferences Just and Carpenter made about mechanisms of encoding negative quantifiers. A related but different explanation is put forward to explain the present data. The experiments show a quantifier function beyond the simple denotation of amount.
\end{abstract}

Psychological work on quantifiers has tended to follow two lines (Sanford, Moxey, \& Paterson, 1994). The classical quantifiers all, some, and no(ne) have been studied largely within the framework of syllogism comprehension (e.g., Evans, Newstead, \& Byrne, 1993; Johnson-Laird, 1983). However, the vast bulk of quantifiers - words such as few, many, a lot, and so on - have been studied in the main with respect to the numbers or proportions they denote. Although it is possible for subjects to indicate what proportion of things quantifiers seem to denote, it seems unlikely that such denotations could be their only function. For instance, if they were differentiated only with respect to numerical denotations, then multidimensional scaling of similarity and confusion matrices for quantifiers should yield unidimensional solutions. Recent work shows that they do not (Routh, 1994), despite early evidence suggesting that they might (Holyoak \& Glass, 1978). Furthermore, the intrasubject variability in estimates of numerical correspondence is so great that it is hard to see how a unidimensional model of quantifier meaning could be viable (see, e.g., Bass, Cascio, \& O'Connor, 1974). By the same token, it would be hard to explain the large variety of quantifying expressions that occur in a language (see

L.M.M. wishes to thank the British Academy for their support of her program of work on quantification, and K.B.P. wishes to thank the Carnegie Foundation for support through a research scholarship. We also wish to thank $M$. Steedman for raising the generalization issue, and J. Hoeksema, H. Kamp, and F. Zwarts for useful discussions and encouragement. Especial thanks go to J. Armstrong, who helped collect the data for Experiments 1 and 2. Correspondence should be addressed to A. J. Sanford, University of Glasgow, Glasgow G12 9YR, Scotland (e-mail: tony@psy.gla.ac.uk).
Moxey \& Sanford, 1993a, for a review of these and related points).

In the present paper, we report three experiments that test and demonstrate the claim that quantifiers may be differentiated through a property of focus. We define differences in focus as differences in the availability of the various subsets that constitute the logical representation of quantified statements. We argue that this is one important aspect of quantifier meaning; it plays a role in the choice of quantifier during production and influences the patterns of activity underlying understanding discourse containing quantifiers. The present experiments build on observations reported in Moxey and Sanford (1987, 1993a).

The idea is illustrated in Figure 1. Quantified statements typically require a number of subsets to be taken into consideration in their definition, and this is the basis of the focus argument. Consider the simple quantified statement, Some of the football fans went to the game. The three sets that need to be taken into account during logical reasoning are shown in Figure 1 and are as follows:

Set 1 , the reference set: a necessary nonempty set of fans who went to the game.

Set 2, the complement set: a possible set of fans who did not go to the game. This is the complement of Set 1 .

Set 3, a possible set of people who went to the game but were not fans. We do not discuss this set in the present paper.

The other set of interest, the total set of fans, is simply $1+2$.

We claim that, although all of these sets have to be taken into account in logical reasoning (with syllogisms, for instance), they are not equally accessible, and that 


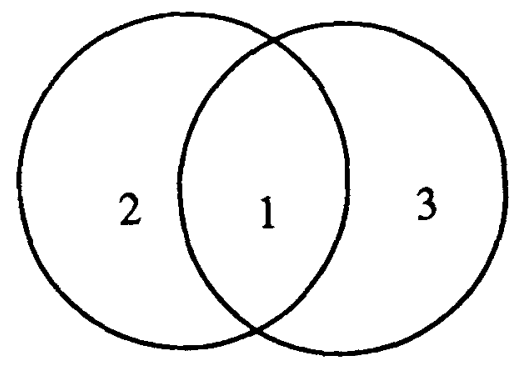

Figure 1. Simple set diagram for representing a quantified sentence of the form Some As are Bs.

with the quantifier some, the most accessible set is Set 1 , the set of fans who went to the game, which we term reference set. This may be illustrated by making an intuitive judgment about the subset in Figure 1 to which a pronominal anaphoric reference is most easily made:

(1) Some of the football fans went to the game.

(1) They watched it with enthusiasm.

Here they refers to the fans who went to the game, that is, the reference set. If the pronoun refers to the fans who did not go to the game - the complement set, as in ( $\left.2^{\prime}\right)$ the reference is less acceptable, or not acceptable at all:

(2) Some of the football fans went to the game.

$\left(2^{\prime}\right)$ They watched it on TV instead.

Indeed, if we wish to refer to the fans who did not go to the game, it seems best to mention them explicitly:

(3) Some of the football fans went to the match.

(3') Those who did not watched it on TV instead.

Moxey and Sanford (1987, 1993a) observed that some quantifiers, specifically those that are negative (to be defined later), appear to license or encourage reference to Set 2, the complement set. For instance, consider substituting few for some in the example discussed above:

(4) Few of the football fans went to the game.

(4') They watched it on TV instead.

$\left(4^{\prime \prime}\right)$ They enjoyed it very much.

In $\left(4^{\prime}\right)$, the pronoun refers to fans who did not go to the game, that is, Set 2, the complement set. An alternative reference set continuation, like ( $\left.4^{\prime \prime}\right)$, seems less acceptable, although not as bad as $\left(2^{\prime}\right)$ is with respect to (2). Anecdotal observation suggests that this reference pattern is restricted to quantifiers that have a semantically negative component, which is either explicit (as in not many) or implicit (as in few). We shall discuss negativity shortly.

In the first two experiments, we investigate focus patterns by using a continuation procedure in which subjects write continuations to quantified sentences starting with the pronoun They. Ease of pronominal reference has been used to make claims about which aspects of discourse lead to something being in focus, roughly meaning being an accessible or prominent part of the mental representation of a discourse (e.g., Ariel, 1990; Chafe, 1972; Sanford \& Garrod, 1981). Examples of focusing properties reported include the antecedent of a pronoun (1) being the agent of a sentence (Hudson, Tannenhaus, \& Dell, 1986), (2) being mentioned early in a sentence (Gernsbacher, 1989), or (3) being denoted by a proper name rather than a simple noun phrase (Sanford, Moar, \& Garrod, 1988). In the present paper, we also assume that ease of pronominal reference is an index of how available for reference a particular subset is. The claim that ease of pronominal reference reflects the dominance or degree of focus of a representation is well motivated, since a nominal pronoun has very little information content (gender, number, reflexivity). Thus, successful resolution of pronominal reference must often rely on other constraints, such as the salience or dominance of a representation to help identify the correct antecedent.

The examples of focus shown so far are anecdotal in nature. However, Moxey and Sanford (1987) obtained data from a large number of subjects who were presented with sentences like (4) and asked to continue with a further sentence, maintaining coherence, and starting with the pronoun They. The negative quantifiers not many, few, and very few led to a pattern in which more than half of the continuations were to fans who did not attend the game. In contrast, a few (and many), both positive, did not allow these continuations; virtually all continuations were of the reference set variety. Moxey and Sanford concluded that negative quantifiers license (allow) but do not require complement set reference patterns, whereas positive quantifiers require the reference set pattern and block the complement set pattern. Complement set focus occurs with negative quantifiers, but this bias appears to be somewhat weaker than the reference set focus bias with positive quantifiers.

The remainder of this paper consists of three studies that extend what is known about these focus effects in a number of ways. The first two experiments expand the range of quantifiers explored, again using a continuationtask method, but with a broad sampling of stimulus materials. Moxey and Sanford (1987, 1993a) used only two basic situations in order to demonstrate the existence of complement set references. These two experiments enable us to distinguish between two alternative accounts of complement set focus, as well as providing a more thorough test of the conditions under which different focus patterns occur. Experiment 3 tests the prediction that focus patterns should be revealed in self-paced reading time, reflecting comprehension processes, as well as in production.

\section{THE NATURE AND LIMTTS OF THE FOCUS EFFECT}

The first two experiments were designed to manipulate two factors: the size of the proportion normally denoted by an expression, and whether or not the expression is considered negative or positive. 


\section{Generalizations and Scale Position}

Quantifiers may denote proportions that vary from almost $0 \%$ (e.g., hardly, any) through to almost $100 \%$ (e.g., almost all). In the original study by Moxey and Sanford (1987), only quantifiers denoting proportions near $0 \%$ were examined. In fact, it was supposed that complement set focusing might well be restricted to quantifiers that denote small proportions. In Experiments 2 and 3, we examine the possibility that quantifiers denoting proportions close to $100 \%$ might also be complement set focusing, provided they are negative. (A quantifier denoting a proportion near $100 \%$, such as nearly all, specifies a reference set containing near $100 \%$, leaving a complement set close to $0 \%$ ). At one level, this study simply increases our knowledge of the conditions under which complement set focus occurs. However, more than this, the scale position question impinges on different conceptions of what complement set focus might be.

A distinction can be made between the generalization and the true complement set focus accounts of the phenomenon. To understand the generalization account, consider the following example of reference:

(5) Few of the senators went to the meeting.

$\left(5^{\prime}\right)$ They stayed at home and watched sports on TV instead.

Sentence $\left(5^{\prime}\right)$ might be considered as being a reference to senators in general, in which case it could be paraphrased as follows:

$\left(5^{\prime \prime}\right)$ In general they stayed at home and watched sports on TV instead.

This is not an unreasonable idea, since sometimes it might be more desirable to refer to what is generally true of the superset rather than what is true of the minority reference set. The complement set character of this example arises from the fact that if a senator stays at home instead, then he/she must be a member of the complement set. But the fact remains that the assertion could be about senators in general.

We suggest that if the complement set is a small (less than $50 \%$ ) proportion of the total set, the generalization argument cannot hold for cases like (5) above. If most senators went to the meeting, any reference to those who didn't go because they went somewhere else instead could not possibly be a generalization. Instead, it would have to be a genuine complement set reference. Indeed, with an example like (5), this should be true if the complement set is less than $50 \%$. If the complement set pattern observed with quantifiers denoting small proportions occurs because people are making whole set or generalization statements, it may be the case that the complement set pattern would not occur at all with quantifiers denoting high proportions.

\section{Negativity}

The condition that licenses complement set references is that the quantifier in question is negative (Moxey \&
Sanford, 1993a). Here we discuss criteria for classing quantifiers as negative for the purposes of laying the ground for the experiments.

Some negatives have a manifest negative component, such as not in not many, but in other cases, the negativity is implicit, as in few. Klima (1964) discussed a number of tests that address different aspects of negativity. One way of determining whether a quantifier is negative is by using constructions that require a negative term in the sentence in which they appear. There exists a class of item, termed negative polarity items, that have been supposed to occur only in the environment of a negative expression (see van der Wouden, 1994; Zwarts, 1994, for logico-linguistic discussions). An example of such an item is anymore: Note that anymore is licensed by a simple declarative sentence in (6) where the quantifier choices are negative, and blocked in (7) where they are positive:

(6) No students/Few students like jazz anymore.

(7) All students/A few students like jazz anymore.

There are many different classes of word that are negative polarity items; examples include quite complex expressions such as "give an X," so, John didn't give a damn is acceptable, but John gave a damn is not (except to produce a special effect). Negative polarity items can be used in this way as a test for negativity, and all items claimed as negative in the present paper pass this test. For instance, the expressions not quite all and less than $100 \%$, which denote proportions close to $100 \%$, are negative:

(8) Not quite all/ Less than $100 \%$ of the students like psychology anymore.

\section{EXPERIMENT 1 Focus Over a Wide Range of Denotations}

\section{Method}

Design and Materials. The conditions $(2 \times 5)$ consisted of negative versus positive quantifiers (2) with 5 quantifiers covering a range of denotations. This required 10 quantifiers in all, as depicted in Table 1 . They were chosen to span the high, low, and middle of the range of proportions a quantifier can denote. As a pretest, 10 subjects were asked to indicate their best estimate of the proportions denoted by each quantifier in a single sentence frame, as illustrated by (9):

\section{(9) Not many of the students were at the meeting.}

Although it is known that the numbers people produce for a given quantifier under these circumstances are a function of the base rate expectation associated with the context, rank order is fairly well preserved over different contexts (Moxey \& Sanford, 1993a). The results of this pretest, given in Table 1 , show the validity of the crude tripartite classification. The distinction between negative and positive was based on the polarity test described above; those items that licensed the use of anymore were taken as negative and those that did not were taken as positive.

The materials were based on a set of 30 sentences, shown in Appendix A. A typical example is:

QUANT of the students produced good essays. 
Table 1 Quantifiers Used in Experiment 1

\begin{tabular}{lll}
\hline & \multicolumn{1}{c}{ Positive } & \multicolumn{1}{c}{ Polarity } \\
Pesition & A few $(15 \%)$ & $\begin{array}{l}\text { Few }(13 \%) \\
\text { Not many }(15 \%)\end{array}$ \\
Mid-to-high & $\begin{array}{l}\text { More than half }(60 \%) \\
\text { Many }(65 \%)\end{array}$ & $\begin{array}{l}\text { Less than half }(37 \%) \\
\text { Near-to-all }\end{array}$ \\
& $\begin{array}{l}\text { Almost all }(95 \%) \\
\text { Nearly all }(95 \%)\end{array}$ & Not all $(80 \%)$ \\
\hline
\end{tabular}

Note-The numbers indicate best estimates of the proportions that each quantifier denotes (i.e., its reference set proportion), taken from the pretest. All polarity claims are based on the materials licensing the use of negative polarity items in a declarative context.

In the full set of experimental materials, each quantifier was used to replace $Q U A N T$ in each of the 30 basic sentence frames, creating 300 items in all.

Each sentence was typed on a separate sheet, in the following form, with a plural pronoun following:

Few of the parents allowed their children to the club. They...

Each subject saw only one quantifier/material combination and thus had to make only a single judgment. This procedure allowed the use of the referent-checking procedure described in the Procedure section, and prevented contrast effects from biasing the results.

Subjects. The 300 subjects were first-year undergraduates naive about the experiment and about psycholinguistics in general. Each subject was allocated one of the sheets at random. Thus 30 subjects responded to any 1 quantifier (cell of the design).

Procedure. Subjects were told to look at the sentence on the sheet and to complete the sentence starting "They..." so that the continuation made sense. They were asked not to turn over their sheets until told. They were monitored to ensure that they conformed to this request. When the sentence had been completed, they were asked to turn over the sheet. On the side they turned to, they found a question asking them to check one of a list of possible referents for the pronoun they that they thought best fitted their intention. The five options for the example material above were
(1) the parents who allowed their children to go, (2) the parents in general, (3) the parents who did not allow their children to go, (4) all of the parents, and (5) other. If (5) was chosen, the subject was asked to specify further. The question was tailored appropriately to each sentence, but the referential possibilities always covered Types $1-5$. Note that (2) and (4) are not strictly equivalent, but that either might be checked if the continuation sentence portrayed a generalization. Having the subjects check the referent served as an index of what the subjects thought they were talking about in the continuation, and as a cross-check in the event of continuations that might be hard to classify.

\section{Results}

The continuations were examined by three independent judges who decided whether the category checked by the subject seemed to be reasonable, in which case the category produced by the subject was recorded. In the event of a disagreement with the subject by any judge, the continuation was treated as unclassifiable. This happened in only 5 cases in Experiments 1 and 2 together. The judges also classed the content of continuations with respect to type, as discussed below.

Reference class. The distribution of response types by quantifier is shown in Table 2 . It is strikingly clear that although the positive quantifiers produced almost all reference set continuations ( $93 \%$ ), and no complement set references whatsoever, the negative quantifiers produced a spectrum of reference types, including a high proportion of complement set references ( $71 \%$ overall). This confirms the association of complement set with negative quantifiers and the blocking of the complement set pattern by positive quantifiers. The low incidence of in general and all continuations (8\%) is consistent with previous observations that subjects tend to see their continuations as complement sets and not as generalizations, in the case of continuations to the negative quantifiers, and as reference sets in their continuations to the positive quantifiers. The occurrence of $15 \%$ reference set continuations in responses to the negative expressions confirms the asym-

Table 2

Reference Patterns From Experiment 1

\begin{tabular}{lcccccc}
\hline \multicolumn{1}{c}{ Quantifier } & \multicolumn{5}{c}{ Reference Classes } & \\
\cline { 2 - 7 } & Reference & Complement & General & All & Other & Unclassified \\
\hline Not quite all & 4 & 19 & 4 & $2(6)$ & 1 & 0 \\
Not all & 2 & 24 & 0 & $2(2)$ & 1 & 1 \\
Less than half & 7 & 18 & 0 & $2(2)$ & 0 & 1 \\
Not many & 2 & 25 & 1 & $1(2)$ & 1 & 0 \\
Few & 8 & 20 & 0 & 0 & 1 & 1 \\
Overall negative & $15 \%$ & $71 \%$ & $3 \%$ & $5 \%(8 \%)$ & $3 \%$ & $2 \%$ \\
Nearly all & 29 & 0 & 1 & $0(1)$ & 0 & 0 \\
Almost all & 27 & 0 & 2 & $1(3)$ & 0 & 0 \\
More than half & 25 & 0 & 2 & $2(4)$ & 1 & 0 \\
Many & 30 & 0 & 0 & 0 & 0 & 0 \\
A few & 29 & 0 & 0 & 0 & 0 & 1 \\
Overall positive & $93 \%$ & $0 \%$ & $3 \%$ & $2 \%(5 \%)$ & $1 \%$ & $1 \%$ \\
\hline
\end{tabular}

Note-The Unclassified column shows materials that resulted from a judge finding the subject's continuation categorization unintelligible, or where the subject failed to check a continuation option. Other classes as defined in the text. The numbers and percentages in parentheses represent the combined value for all and in general, and correspond to the generalization class. 
metry of the focus properties of the negatives, which are somewhat diffuse, and the positives, which appear to block complement set references completely. An overall chi-square test between pooled negative and positive quantifiers using the summary frequencies of the reference classes reference set, complement set, and pooled general/ all was highly reliable $\left[\chi^{2}(2)=171.54, p<.001\right]$. The use of chi-square was motivated by the fact that a given subject provided only one response to the data pool.

Although there is some numerical variability of the incidence of complement sets over the three levels of denotation, the difference is not reliable. A chi-square test was carried out on the frequencies of complement set continuations for the negative quantifiers, and it showed no reliable effect $\left[\chi^{2}(4)=1.83\right.$, n.s.]. There are thus as many complement set references to sentences based on quantifiers denoting proportions that are high as to those denoting proportions that are low or intermediate.

The content of the continuations. The judges classified each and every continuation according to a schema devised by Moxey and Sanford (1987) designed to capture the principal types of continuation. These are shown below, along with a test for each classification:

Reason-not: The continuation provides a reason that contradicts or does not support the behavior described in the predicate. For instance: Few of the football fans went to the game. They watched it on TV instead. The continuation provides a reason why some of the fans were not at the game.

Test: Ask whether Fans didn't go to the match because CONTINUATION is acceptable.

Reason-true: The continuation provides a reason that supports the behavior described in the predicate. For instance: More than half of the football fans went to the game. They thought it would be a really good game. The continuation provides a reason for going to the match.

Test: Ask whether Fans went to the game because $C O N$ TINUATION is acceptable.
Consequence (of number): The continuation describes something that is a consequence of the number of people whose behavior fits the predicate. For instance: Not many of the football fans went to the game. They had to cheer really loudly to make themselves heard.

Test: Ask whether CONTINUATION because QUANT of fans went to the game is acceptable.

Other: None of the other categories seem to fit. This includes "simple" continuation, which simply says what happened next and does not indicate anything about reasons and consequences. For instance: $A$ few of the football fans went to the game. They got wet when it started to rain.

In the event of ultimate disagreement, the material could be left out of the classification and shown as Unclassified in Table 3; in practice this very rarely happened. Continuations to negative and positive quantifiers differ considerably. The dominant response class for negatives is Reason-not, whereas none of this class occur with the positives $\left[\chi^{2}(1)=63.2, p<.001\right]$. In contrast, the dominant type of response to positive quantifiers was Other; these responses also occurred with negatives, but to a lesser extent, with the difference being reliable $\left[\chi^{2}(1)=\right.$ $9.19, p<.001]$. The second most dominant response type to positives was Reason-true; this category occurred very much less often in the case of negatives $\left[\chi^{2}(1)=\right.$ $33.1, p<.001]$. The principal response type to negatives is thus the statement of a reason, cause, or supporting evidence as to why the predicate does not hold for some members of the quantified set (i.e., the complement set).

Exclusive set continuations. The complement set continuations were examined for special, highly discriminating cases that were unequivocally what we shall term exclusive sets, as illustrated by (10):

(10) Not quite all of the football fans went to the game. They were drunk and lay unconscious on the pavement.

Table 3

Continuation Types From Experiment 1

\begin{tabular}{lccccc}
\hline & \multicolumn{5}{c}{ Continuation Types } \\
\cline { 2 - 6 } \multicolumn{1}{c}{ Quantifier } & Reason True & Reason Not & Consequence & Other & Unclassified \\
\hline Not quite all & 3 & 15 & 0 & 12 & 0 \\
Not all & 1 & 20 & 0 & 8 & 1 \\
Less than half & 3 & 16 & 0 & 10 & 1 \\
Not many & 1 & 19 & 0 & 10 & 0 \\
Few & 1 & 12 & 0 & 16 & 1 \\
Overall negative & $6 \%$ & $55 \%$ & $0 \%$ & $37 \%$ & $2 \%$ \\
Nearly all & 11 & 0 & 0 & 19 & 0 \\
Almost all & 15 & 0 & 0 & 15 & 0 \\
More than half & 13 & 0 & 1 & 16 & 0 \\
Many & 8 & 0 & 0 & 22 & 0 \\
A few & 8 & 0 & 0 & 21 & 1 \\
Overall positive & $37 \%$ & $0 \%$ & $1 \%$ & $61 \%$ & $1 \%$ \\
\hline
\end{tabular}

Note-Unclassified refers to continuations where there was disagreement between the judges, or where there was no continuation produced by the subject. Other categories as defined in the text. 
Unequivocally, being unconscious on the pavement precludes being at the game; it excludes the reference set, and so there is no doubt that the pronoun they refers to the complement set proper. For not all, 15/22 cases $(68 \%)$ of reported complement set reference fell into this category, whereas for not quite all, there were $11 / 18$ cases $(61 \%)$. Thus for the quantifiers whose reference set was near $100 \%$, more than half of the continuations classified as complement set by the subjects were indeed necessarily true complement set references. This is perhaps the strictest test of complement set reference in the sense of the set diagram shown in Figure 1.

\section{Discussion}

Negative quantifiers produce a high rate of reported complement set references, averaging $71 \%$ of all responses, and a much lower rate of reported generalizations, averaging $8 \%$ of all responses. Furthermore, reported complement set references occur with essentially undifferentiated likelihoods for all quantifiers. There is no evidence that they occur at reduced levels for quantifiers denoting high proportions. An analysis of continuations for negative quantifiers shows a relatively high proportion of the Reason-not types. We shall explore this more fully in the General Discussion. When the Reasonnot responses are further broken down into exclusive set continuations for the quantifiers denoting large proportions, $65 \%$ of continuations reported as complement set were indeed necessary complement set references. Although it is possible that some continuations are generalizations and yet reported as complement sets, it is evident that complement set reference proper does occur.

In Experiment 2, a similar test was carried out on another group of negative and positive expressions.

\section{EXPERIMENT 2}

\section{Homogeneous Expressions}

The purpose of Experiment 2 was to provide a further check on the observation that complement set focus oc- curs with negatives over a full range of proportion denotations. In Experiment 1, scale effects were confounded with quantifier type, some expressions being simple and others complex. In the present experiment, advantage was taken of the fact that the modifiers nearly and not quite form expressions that are positive and negative; they also take arguments that in principle may be anywhere in the range of near $0 \%$ to $100 \%$. The questions are as follows: Does the complement set pattern reveal itself with these expressions? Does it occur over the full range? Does it show any variability over the range?

\section{Method}

Materials and Design. The scenarios used were the same as those in Experiment 2. Each of the 30 scenarios was used with the following expressions substituted for $Q U A N T$ : the negative not quite followed by $10 \%, 30 \%, 50 \%, 70 \%, 90 \%$; and the positive nearly followed by $10 \%, 30 \%, 50 \%, 70 \%, 90 \%$. The design was thus 2 (negative/positive quantifier) $\times 5$ (proportion denoted).

Procedure. The procedure was the same as that in Experiment 1. The continuation question was on one side of the sheet given to the subjects, and the query regarding pronominal reference was on the other side. Each subject saw only 1 sheet.

Subjects. There were 300 volunteer subjects, all from introductory classes in psychology. The students had no knowledge of the hypotheses under test.

\section{Results}

The continuations were classified in exactly the same way as in Experiment 1.

Reference class. The proportions of reference types for each quantifier are shown in Table 4 . The positive quantifiers produced only one complement set reference in total, and a predominance of reference set references $(87 \%)$. Eleven percent of the continuations were of the all/generalization type. In contrast, the negative quantifiers produced a spectrum of reference types, including complement set references ( $53 \%$ overall). Generalizations amounted to $11 \%$. Reference set continuations were the second largest class, at $27 \%$, again showing the more diffuse nature of the focus pattern in the case of the neg-

Table 4

Reference Types for Experiment 2

\begin{tabular}{|c|c|c|c|c|c|c|}
\hline \multirow[b]{2}{*}{ Quantifier } & \multicolumn{6}{|c|}{ Reference Classes } \\
\hline & Reference & Complement & General & All & Other & Unclassified \\
\hline Not quite $10 \%$ & 9 & 15 & 1 & $3(4)$ & 2 & 0 \\
\hline Not quite $30 \%$ & 8 & 18 & 1 & $1(2)$ & 2 & 0 \\
\hline Not quite $50 \%$ & 2 & 23 & 2 & $1(3)$ & 2 & 0 \\
\hline Not quite $70 \%$ & 12 & 13 & 2 & $0(2)$ & 1 & 2 \\
\hline Not quite $90 \%$ & 12 & 10 & 3 & $2(5)$ & 0 & 3 \\
\hline Overall negative & $27 \%$ & $53 \%$ & $6 \%$ & $5 \%(11 \%)$ & $5 \%$ & $3 \%$ \\
\hline Nearly $10 \%$ & 25 & 1 & 3 & $1(4)$ & 0 & 0 \\
\hline Nearly $30 \%$ & 27 & 0 & 0 & $1(1)$ & 1 & 1 \\
\hline Nearly $50 \%$ & 27 & 0 & 1 & $1(2)$ & 1 & 1 \\
\hline Nearly $70 \%$ & 25 & 0 & 5 & $0(5)$ & 0 & 0 \\
\hline Nearly $90 \%$ & 26 & 0 & 1 & $3(4)$ & 0 & 0 \\
\hline Overall positive & $87 \%$ & $1 \%$ & $7 \%$ & $4 \%(11 \%)$ & $1 \%$ & $1 \%$ \\
\hline
\end{tabular}

Note-The numbers and percentages in parentheses represent the combined values for all and in general, and correspond to the generalization class. 
Table 5

Continuation Types for Experiment 2

\begin{tabular}{lcccccc}
\hline & \multicolumn{5}{c}{ Continuation Types } \\
\cline { 2 - 4 } Quantifier & Reason-true & Reason-not & Consequence & Other & Unclassified \\
\hline Not quite $10 \%$ & 4 & 15 & 0 & 11 & 0 \\
Not quite $30 \%$ & 2 & 15 & 0 & 13 & 0 \\
Not quite 50\% & 0 & 17 & 0 & 13 & 0 \\
Not quite 70\% & 4 & 10 & 0 & 12 & 4 \\
Not quite $90 \%$ & 10 & 9 & 1 & 8 & 2 \\
Overall negative & $13 \%$ & $44 \%$ & $.7 \%$ & $38 \%$ & $4 \%$ \\
Nearly 10\% & 13 & 0 & 1 & 16 & 0 \\
Nearly 30\% & 12 & 0 & 0 & 17 & 1 \\
Nearly 50\% & 12 & 1 & 1 & 16 & 0 \\
Nearly 70\% & 12 & 0 & 0 & 18 & 0 \\
Nearly 90\% & 10 & 0 & 0 & 20 & 0 \\
Overall positive & $39 \%$ & $.7 \%$ & $1.3 \%$ & $58 \%$ & $.7 \%$ \\
\hline
\end{tabular}

atives. An overall chi-square test on pooled responses to negatives versus positives, using the classes reference set, complement set, and all + in general was significant $\left[\chi^{2}(2)=105.1, p<.001\right]$.

The incidence of complement set references showed some numerical variation over proportion, being maximal in the midrange, but a chi-square test of complement set incidence over negative quantifiers did not yield a reliable result $\left[\chi^{2}(4)=6.25\right.$, n.s. $]$.

Continuation types. Table 5 details the continuation types, which were based on the same classification as that used in Experiment 1. In contrast to the findings of Experiment 1 , the Other class dominated continuations with both the negative ( $43 \%)$ and positive $(58 \%)$ quantifiers: The difference between them was not reliable $\left[\chi^{2}(1)=3.18, p<.1\right]$. Reason-not types occurred as frequently as Other types in the negatives, and this rate was much higher at $66 \%$ than it was for the positives, where it occurred on only one occasion $\left[\chi^{2}(1)=48.5, p<\right.$ $.001]$. In contrast, Reason-true responses accounted for $39 \%$ of continuations to positives, and only $13 \%$ to negatives $\left[\chi^{2}(1)=19.3, p<.001\right]$.

Exclusive set continuations. There were 14 exclusive set continuations for the 22 complement set continuations to not quite $70 \%$ and not quite $90 \%$, further evidence of clear complement set reference.

\section{Discussion}

The incidence of complement set continuations was slightly lower in Experiment 2 than in Experiment 1 ( $53 \%$ vs. $71 \%$ ), and a chi-square test on the relative complement set rates was just reliable $\left[\chi^{2}(1)=3.94, p<\right.$ $.05]$. There was also a difference between the number of Reason-not type continuations in the two experiments (55\% in Experiment 1 vs. 39\% in Experiment 2), but this failed to reach significance $\left[\chi^{2}(1)=3.75\right.$, n.s.]. Why these differences hold is not clear; it may be that the use of percentages resulted in weaker focus effects. However, in general, the pattern of Experiment 1 was reproduced here: Complement set occurred with negatives regardless of the proportion the quantifier denoted, and negatives produced a more diffuse focus pattern than positives, which effectively blocked complement set reference. Complement set focus is licensed by negative quantifiers, but reference set and generalizations are allowed. In fact, reference set references occurred with negatives on 15\% of occasions in Experiment 1 and 27\% in Experiment 2. On the other hand, for positives, complement set focus was blocked. Reference set and generalizations were licensed but reference set prevailed.

The relative flexibility associated with negative quantifiers raises the question of whether focus pattern with these expressions would have any impact on the processes of integration during reading. In contrast, we would certainly predict that focus pattern would play a role in integration in the positive case. This is addressed in Experiment 3.

\section{EXPERIMENT 3 Reading Time Measures}

The focus effects associated with negativity have been demonstrated only with the continuation procedure, an off-line production task. In Experiment 3, we attempted to extend the demonstration of focus differences by using self-paced reading time. If focus is set up by a quantified sentence, a subsequent sentence that does not fit the preferred focus pattern should be disadvantaged in processing.

Consider the material in Table 6. In terms of the biases suggested by the continuation studies, the combination $a$ few/Their presence corresponds to reference to the set who attended the meeting (i.e., the set of which the predicate of the quantified sentence is true), which is a com-

Table 6

A Typical Item From Experiment 3

\section{A Public Meeting}

Local MPs were invited to take part in a public enquiry about proposals to build a new nuclear power station. A few/few* of the MPs attended the meeting. Their presence/absence* helped the meeting run smoothly.

Was the public enquiry about sewage disposal?

*The slashes denote options. 
patible quantifier/reference transition; the transition $a$ few/Their absence is incompatible, since Their absence refers to members of the complement set, and $a$ few should block complement set focus. The time to process sentences based on the incompatible transition should be longer. In the case of the combination few/Their presence, provided the complement set focus dominates, this transition too might be classed as incompatible. However, given the spontaneous occurrence of reference set continuations, it is possible that this transition would be less disruptive, or even not show up at all if negatives allow both focus patterns. Thus we might predict some sort of asymmetry between the mismatch effects for positive and negative quantifiers: Mismatches with the negative might yield a smaller difference or no difference at all.

\section{Method}

Materials. A total of 24 snippets of text were created, and these are listed in Appendix B. Table 6 gives a typical example. In each case the possessive phrases in the third sentence (e.g., their presence/ absence, their strictness/laxity) allowed compatible or incompatible transitions. The materials were arranged for display one sentence at a time. Although the first sentence sometimes ran to more than one line, the second sentence (introducing the quantified statement) appeared in a single (separate) line, as did the third (target) sentence. Each item ended with a question designed to guarantee that the subject attended to what she/he was reading. For 12 of the items, the answer to the question was "yes" and for 12 it was "no."

Design. The design had one between-subjects variable: whether the quantifier comparison was between few and a few, or between not many and many. Two separate groups of subjects saw items using these quantifier pairings. Within each subgroup, a particular quantifier was followed by a target sentence with either a compatible or an incompatible anaphoric reference sentence following, as defined earlier. This produced 4 within-subject conditions:

Condition 1: a few or many + reference set reference;

Condition 2: a few or many + complement set reference;

Condition 3: few or not many + reference set reference; and

Condition 4: few or not many + complement set reference.

The stimuli were presented in matched random order, so that if an item in one form appeared in (say) third position, in a file, there were three other matching files corresponding to the material in the other forms. In addition to the 24 experimental items, 24 filler items were used, which were similar multisentence texts but that did not consist of quantifier focus manipulations.

Procedure. Subjects were seated in front of a black-and-white monitor linked to a Macintosh IIsi computer running PsyScope software (Cohen, McWhinney, Flatt, \& Provost, 1993). Subjects were tested individually. They were presented with each passage in turn and were asked to press a button centrally mounted on a button box to observe each sentence of the passage. They were told that at the end of each passage, a yes/no question would be asked. They were told to press the red (left) button if the answer was yes, and the green (right) button if the answer was no. Subjects were instructed on keeping their fingers comfortably positioned on the keys, ready for fast responses. They were told to read as quickly as was compatible with understanding and to answer the questions as quickly and accurately as possible. At the end of each passage, a fixation spot appeared for $1.5 \mathrm{sec}$ in the top left corner of the screen. This was automatically replaced by the passage title, and subjects then called up the next sentence at their own pace. The PsyScope software recorded response times with millisecond ac- curacy and recorded the response type to the questions. Prior to the experiment proper, the subjects were given 5 practice items.

Subjects. There were 48 unpaid volunteer subjects, 24 being assigned randomly to each subgroup. All were students attending an introductory summer school at the University of Glasgow, with normal or corrected-to-normal eyesight, and they were unaware of the aims of the experiment.

\section{Results and Discussion}

Error rate. Error rates on questions averaged 10\%, which was considered low enough to indicate that subjects were trying to understand what they were reading. Rates by condition were as follows: $10.3 \%$ for positive reference set transitions, $10.6 \%$ for positive complement set transitions, $8.9 \%$ for negative reference set transitions, and $10.4 \%$ for negative complement set transitions. These did not differ reliably from one another.

Reading times. Mean reading times were examined for both the critical sentence (anaphoric reference sentence) and the preceding sentence, which introduced the quantifiers. For each condition, outliers were removed by pruning times that fell beyond 2 standard deviations either side of the mean. The resultant mean reading times for the anaphoric reference sentences are shown in Figure 2 . Two $2 \times 2 \times 2$ analyses of variance were carried out on the data, the factors being negative versus positive quantifier, quantifier pair (not many vs. many, or few vs. a few), and anaphoric reference type (complement set vs. reference set). Analyses were carried out by subjects and by items.

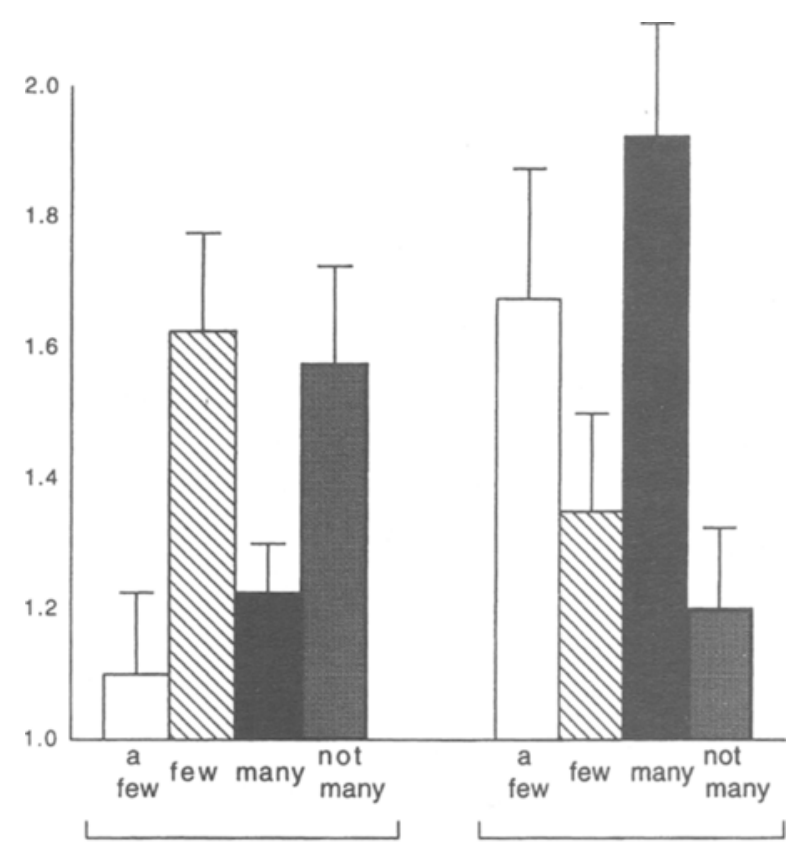

REFERENCESET

COMPLEMENT SET

\section{REFERENCE TYPE IN TARGET SENTENCE}

Figure 2. Reading times in seconds, with standard error bars, for target sentences in Experiment 3. The target sentences contain references that are focused on either the complement set or the reference set. 
The most robust effect was that the reading times for sentences containing complement set references were slow with positive quantifiers and faster with negative quantifiers, whereas those containing reference set references were slower with negative quantifiers and faster with positive quantifiers. This is revealed as an interaction in both the subjects analysis $\left[F_{1}(1,46)=46.55, p<\right.$ $.0001]$ and the item analysis $\left[F_{2}(1,46)=33.19, p<\right.$ .0001 ], giving $\min F^{\prime}(1,47), p<.001$. This confirms the main hypothesis behind the experiment.

Only one main effect approached significance: Complement set focus reading times were slower by some $230 \mathrm{msec}$, an effect that was significant by items $\left[F_{2}(1,46)\right.$ $=6.66, p<.013]$, but that was not quite significant by subjects $\left[F_{1}(1,46)=3.69, p<.061\right]$. There was no main effect of quantifier pair $[F(1,46)=0.03]$, and there was no main effect of negative versus positive quantifier.

No other interaction was significant. It had been conjectured that a complement set pattern following a positive quantifier might be more disruptive than a reference set pattern following a negative quantifier, since the continuation data showed that negatives license complement set but also allow reference set, whereas positives seem to block complement set. However, since there was no interaction between positive/negative quantifier and the magnitude of the mismatch effect, but only a main effect in that complement set is (weakly) slower than reference set, we can only tentatively conclude that complement set anaphors are processed more slowly in general.

Additional 2 (quantifier pair) $\times 2$ (negative vs. positive quantifier) $\times 2$ (subsequent reference type) analyses of variance (ANOVAs) were carried out on reading times for the preceding (quantified) sentence. This was done to see if processing of sentences containing a negative quantifier was slower than processing of sentences containing positive ones (see, e.g., Clark, 1976) and to serve as a control for the main analysis. Mean reading times, shown in Figure 3, reveal some trend for the negatives to be processed more slowly. However, the effect, at $108 \mathrm{msec}$, although reliable by subjects $\left[F_{1}(1,46)=4.90, p<.032\right]$, was not quite reliable by items $\left[F_{2}(1,46)=3.29, p<\right.$ $.08]$. Thus the data suggest, albeit tentatively, that negative quantifiers are perhaps processed more slowly than positive quantifiers. No other effect, main or interaction, approached significance for either $F_{1}$ or $F_{2}$ (all $F \mathrm{~s}<1.9$ ).

The main hypothesis behind Experiment 3 was confirmed: The focus effects previously observed through the analysis of continuations played a role in the integration of successive sentences in text. The quantifiers not many and few, both negative, favor integration of subsequent sentences that have a focal reference to the complement set. In contrast, their positive counterparts many and a few favor integration of subsequent sentences that have focal references to the reference set. Despite the evidence from the continuation task that the focus pattern of the negative quantifiers is more diffuse, this was not reflected in the present experiment. Clearly, the focus effect with negative quantifiers is just as strong as the positive focus as far as integration during reading is concerned.

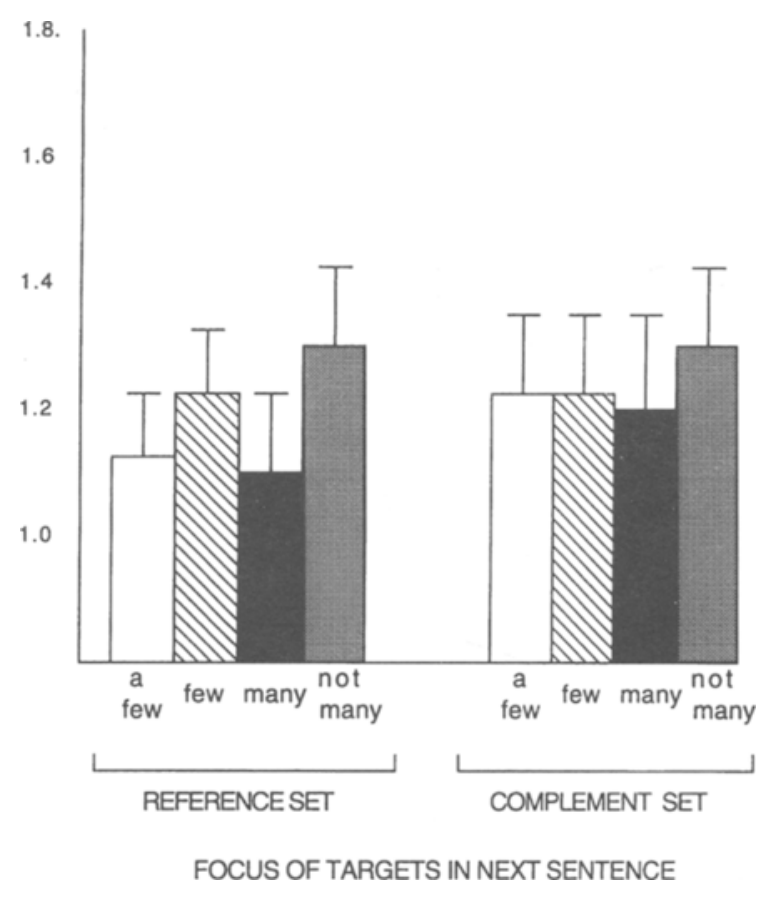

Figure 3. Reading times in seconds, with standard error bars, for the sentences preceding the targets: These contained the quantifiers under investigation. There is a slight tendency for the sentences containing negative quantifiers to be read more slowly.

A final point to note is that the complement set target materials in the present experiment are not, in general, of the Reason-not type that was so prevalent in the responses in Experiments 2 and 3. This means that the match effects were not due to a match of the type of inference expected, but rather to a match in the set being referred to.

\section{GENERAL DISCUSSION}

The results of the present experiments provide corroboration and considerable extension of earlier work (Moxey \& Sanford, 1987; 1993a) showing that negative and positive quantifiers produce different focus effects.

Negative and positive quantifiers serve to put differential focus on the various subsets of the logical model that describes a quantified statement; positive on reference set, and negative on complement set. Although it might be argued that some of the instances of complement set focus may be generalizations, subjects perceive their own acts of reference as being to the complement set. This alone provides the first piece of evidence suggesting that the generalization model of complement set reference might be an oversimplification. A second piece of evidence is an almost total absence (5 cases only) of constructions such as (11), which might be expected if subjects were thinking in terms of generalizations:

(11) Few of the MPs went to the meeting.

They mostly couldn't be bothered.

They preferred doing other things on the whole. 
Also, there was no reduction in complement set reference rate for quantifiers for which the reference set was above $50 \%$ and close to $100 \%$, although one might expect just such a reduction if complement set references are generalizations. Finally, the analysis of exclusive set continuations showed that complement set reference per se is indeed a reality.

Complement set focus can be related to some earlier work. Using a sentence-picture verification task, Just and Carpenter (1971) studied the patterns of verification latencies for quantified sentences. Subjects had to compare statements of the form QUANT of the dots are red with pictures of 16 dots, with variable proportions of red and black dots. For cases where the sentence contained a positive (in our terminology), such as a small proportion of the dots are red, Just and Carpenter found that under the appropriate conditions, subjects encoded the statement in terms of the red dots. In contrast, when the sentence contained a negative, it was inferred from the pattern of matching latencies that subjects were encoding the sentence in terms of the black dots (see also Carpenter \& Just, 1972).

Findings from the present experiments are consistent with this pattern and provide a set of much more direct tests of the idea in a less artificial and restricted context. Clark (1976) saw the matching experiment as fitting a straightforward account of negative processing in which negatives are taken to be coded in terms of what he called their suppositions. Negatives are thought to presuppose that which they deny, and a sentence like Not many of the dots are red is taken as presupposing that there is reason to believe that many of the dots might be red. Such a sentence is supposedly coded as something like (false(many (dots are red)), with a focus on many (see Moxey \& Sanford, 1993b, for a direct test). As a procedure to test a picture, evaluation in terms of what is true of many takes place - and that entails testing the black dots. In effect, this theory says that negative statements are encoded in terms of the complement set. Our results showing complement set focus in production and reading time may be taken as consistent with this account.

However, we would like to suggest a possible alternative. The need for this arises from the apparent asymmetry of the focus patterns of negative and positive quantifiers. Although the positives almost unequivocally ruled out complement set focus, the negatives did not rule out reference set focus: This was particularly evident in Experiment $2(27 \%)$, but was also true in Experiment 1 $(15 \%)$ and in our earlier work (Moxey \& Sanford, 1987). One way to explain the diffuse nature of negative focus would be through a mechanism in which negatives put a processing emphasis on the negated predicate. For instance, given Not many children like ice cream, the processing emphasis goes onto not liking ice cream. By processing emphasis, we mean that inferential activity is anchored on not liking ice cream, which, in a continuation task, could lead to the production of reasons for children not eating ice cream, the consequences of not eating ice cream, things that happened in spite of not eating ice cream, or some other resulting activity consequent on not eating ice cream. By and large, continuations on any of these themes will tend to produce complement set references, because, for instance, reasons for not eating ice cream are more likely to hold for children who do not eat ice cream than for children who do.

Such an account has the potential to explain the diffuse focus pattern found with negatives. It also fits the results of Just and Carpenter's (1971) experiment, because the simplicity of the domain they used does not enable the predicate/set distinction to be made. Thus the sentence Not many of the dots are red could be taken as putting processing emphasis on not being red, which in the dots domain of the experiment perfectly specifies the complement set.

In conclusion, complement set focus is a property associated with negative quantifiers. It is seen to be an influence on comprehension patterns as well as on production (continuation). We anticipate that focus will influence patterns of reasoning that follow from the use of quantified statements. Focus constitutes one way in which quantifiers may be differentiated from one another other than through the notion of them denoting different proportions. Future work will be aimed at a fuller investigation of how it works in real time, as we now know that it has a detectable influence on reading (Experiment 3 ), and should provide further insights into focus asymmetry phenomena.

\section{REFERENCES}

ARIEL, M. (1990). Accessing noun-phrase antecedents. London: Routledge.

Bass, B. M., Cascio, W. F., \& O'Connor, E. J. (1974). Magnitude estimates of frequency and amount. Journal of Applied Psychology, 59, 313-320.

CARPENTER, P. A., \& Just, M. A. (1972). Semantic control of eye movements in picture scanning during sentence-picture verification. Perception \& Psychophysics, 12, 61-64.

ChaFe, W. (1972). Discourse structure and human knowledge. In J. B. Carroll \& R. O. Freedle (Eds.), Language comprehension and the acquisition of knowledge (pp. 41-69). Washington, DC: Winston.

Clark, H. H. (1976). Semantics and comprehension. The Hague: Mouton.

Cohen, J., MacWhinney, B., Flatt, M., \& Provost, J. (1993). PsyScope: An interactive graphic system for designing and controlling experiments in the psychology laboratory using Macintosh computers. Behavior Research Methods, Instruments, \& Computers, 25, 257-271.

Evans, J. St. B., Newstead, S. E., \& Byrne, R. M. (1993). Human reasoning: The psychology of deduction. Hove, U.K.: Erlbaum.

GeRNSBACHER, M. A. (1989). Language comprehension as structure building. Hillsdale, NJ: Erlbaum.

HolyOAK, K. J., \& GLASS, A. L. (1978). Recognition confusions among quantifiers. Journal of Verbal Learning \& Verbal Behavior, 17, 249264.

Hudson, S. B., Tannenhaus, M. K., \& Dell, G. S. (1986). The effect of discourse center on the local coherence of a discourse. In C. Clifton (Ed.), Proceedings of the Eighth Annual Conference of the Cognitive Science Society (pp. 96-101). Hillsdale, NJ: Erlbaum.

Johnson-Laird, P. N. (1983). Mental models. Cambridge: Cambridge University Press.

Just, M. A., \& CARPENTER, P. A. (1971). Comprehension of negation with quantification. Journal of Verbal Learning \& Verbal Behavior, 10, 244-253.

KLIMA, E. S. (1964). Negation in English. In J. A. Fodor \& J. J. Katz 
(Eds.), The structure of language (pp. 246-323). Englewood Cliffs, NJ: Prentice-Hall.

MoXeY, L. M., \& SANFORD, A. J. (1987). Quantifiers and focus. Journal of Semantics, 5, 189-206.

MoXeY, L. M., \& SANFoRD, A. J. (1993a). Communicating quantities: A psychological perspective. Hove, U.K.: Erlbaum.

MoXey, L. M., \& SANFORD, A. J. (1993b). Prior expectation and the interpretation of natural language quantifiers. European Journal of Cognitive Psychology, 5, 73-91.

RouTH, D. A. (1994). On the representation of quantifiers. Journal of Semantics, 11, 199-214.

SANFORD, A. J., \& GARROD, S. C. (1981). Understanding written language. Chichester, U.K.: Wiley.

SANFord, A. J., MoAr, K., \& Garrod, S. C. (1988). Proper names as controllers of discourse focus. Language \& Speech, 31, 43-56.

SAnford, A. J., Moxey, L. M., \& PAterson, K. B. (1994). Psychological studies of quantifiers. Journal of Semantics, 10, 153-170.

VAN DER WoudEN, T. (1994). Negative contexts. Unpublished doctoral dissertation, University of Groningen, The Netherlands.

Zwarts, F. (1994). Polarity items. In R. E. Asher \& J. M. Y. Simpson (Eds.), The encyclopedia of language and linguistics (pp. 32013204). Oxford: Pergamon Press.

\section{APPENDIX A}

Items for Experiments 1 and 2

QUANT of the MPs went to the meeting.

QUANT of the football fans went to the game.

QUANT of the students produced good essays.

QUANT of the soldiers were overweight.

QUANT of the children enjoyed the fair.

QUANT of the hospital patients volunteered for the test.

QUANT of the job applicants did well in the interviews.

QUANT of the accountants took up the retraining offer.

QUANT of the patients liked the new hospital food.

QUANT of the miners believed the new offer on job safety.

QUANT of the athletes trained for two hours each day.

QUANT of the swimmers had good backstroke technique.

QUANT of the schoolboys finished the marathon.

QUANT of the audience clapped when the play finished.

QUANT of the congregation joined in the singing.

QUANT of the parents allowed their children to go to the club.

QUANT of the girl guides missed the swimming practice.

QUANT of the boys on the estate had taken drugs.

QUANT of the couples at the dance could do the new waltz.

QUANT of the teachers supported the candidate for the union.

QUANT of the Wembley crowd cheered the unusual service.

QUANT of the rock-show audience admired the lead guitarist.

QUANT of the purchasers enjoyed the book.

QUANT of the new medical school had been trained in communication skills.

QUANT of the club members came to the AGM.

QUANT of the Indians on the reservation had a healthy diet.

QUANT of the holiday makers lay on the beach.

QUANT of the people on the train slept sometime during the journey.

QUANT of the general airport staff supported the traffic control strike.

QUANT of the parents liked the headmaster's ideas.

\section{APPENDIX B \\ Items for Experiment 3}

The following items illustrate the quantifier manipulation for $a$ few and few. The same items were used for the contrast of many and not many.

Writing an essay.

The literature students were set an essay on Samuel Beckett and modern literary theory. [A few/Few] of the students produced readable results. Their [clarity/confusion] demonstrated how well they understood the topic.

On an identity parade.

Some soldiers from the local barracks were put on an identity parade alongside the accused man. [A few/Few] of the soldiers looked like the accused. Their [differences/similarities] were mentioned by the victim.

At the funfair.

After having a ride on the big wheel a group of college students decided to try the coconut shy. [A few/Few] of the students managed to hit a coconut. Their [accuracy/inaccuracy] earned them a commiseration prize.

In a hospital.

The doctor needed permission from some of the patients before she tested a new drug on them. [A few/Few] of the patients agreed to act as guinea pigs. Their [consent/refusal] was noted by the hospital registrar.

Testing job applicants.

Prospective air traffic controllers had to fill in a personality questionnaire and then sit a series of aptitude tests. [A few/ Few] of the applicants passed. Their [success/failure] confirmed the organizer's expectations.

A job offer.

Some accountancy trainees were offered a job with a major international company. [A few/Few] of the accountants agreed to work for the company. Their [rejection/acceptance] was sent by return of post.

In the court.

Some local youths were arrested during a police raid at a party and accused of drug dealing. [A few/Few] of the youths were found guilty of the crime. Their [acquittal/conviction] was a relief to the whole neighborhood.

Coal mining.

The Coal Board wanted to increase productivity by making changes to working practices. [A few/Few] of the miners believed the promises of job safety. Their [trust/distrust] was the result of a previous deal.

At the gym.

Some weight lifters from the gym competed to see who could lift a heavy dumbbell. [A few/Few] of the weight lifters managed to lift it off the ground. Their [strength/weakness] surprised their friends. 


\section{APPENDIX B (Continued)}

At the swimming pool.

A class of school children had been taking swimming lessons for the last couple of weeks. [A few/Few] of the children could manage the backstroke. Their [incompetence/competence] surprised the instructor.

\section{Getting exam results.}

The girls crowded round the notice board to see the examination results for chemistry and biology. [A few/Few] of the girls did very well. Their [joy/grief] was uncontrollable.

Running a marathon.

Some boys decided to enter the marathon. [A few/Few] of the boys finished the race. Their [unfitness/fitness] surprised their friends.

In the train station.

Passengers often had difficulty finding the platform for the evening train to Birmingham. [A few/Few] of the staff were willing to give directions. Their [helpfulness/unhelpfulness] was reported to the manager.

At the theater.

At the end of the performance the actors came to the front of the stage and took a bow. [A few/Few] of the audience expressed appreciation. Their [silence/applause] was a surprise to the theater manager.

At a church meeting.

The evangelist told the audience that he would cure the sick and disabled among them. [A few/Few] of the audience thought that he could perform miracles. Their [belief/disbelief] was shared by the church authorities.

In the office.

The insurance office had a new computer system installed over the Christmas holidays. [A few/Few] of the secretaries had previously used the system. Their [inexperience/experience] made the others feel more relaxed.

Caught shoplifting.

A group of boys were suspected of stealing magazines and sweets from the newsagents on the corner. [A few/Few] of the boys admitted to the crime. Their [honesty/dishonesty] affected the punishment they received.

\section{APPENDIX B (Continued)}

In the bar.

One of the bar staff liked to joke with female customers when they asked for a drink. [A few/Few] of the women appreciated his sense of humor. Their [displeasure/pleasure] was obvious to everyone.

In a restaurant.

The office party went to an Italian restaurant for their Christmas dinner. [A few/Few] of the office staff treated the waiter with respect. Their [courtesy/rudeness] was reported to the office manager.

Going to a party.

The girls asked their parents if they could go to an all-night party at a classmate's house. [A few/Few] of the parents allowed their daughter to go. Their [laxness/strictness] reflected their own upbringing.

A public meeting.

Local MPs were invited to take part in a public inquiry about proposals to build a nuclear power station. [A few/Few] of the MPs attended the meeting. Their [presence/absence] helped the meeting run more smoothly.

At a Boy Scout camp.

It was dark before the Boy Scouts finished putting up the tents and collected wood for a campfire. [A few/Few] of the scouts went to bed immediately. Their [sleepiness/wakefulness] annoyed the scoutmaster.

In a classroom.

The trainee mathematics teacher was nervous about giving her first unsupervised lesson in such a rough school. [A few/Few] of the class misbehaved. Their [noisiness/quietness] was reported to the real teacher.

At the football match.

The stadium was packed to see Falkirk play East Stirling in the final of the Stirlingshire Cup. [A few/Few] of the Falkirk fans expected Falkirk to win. Their [optimism/pessimism] was rewarded by an early goal.

(Manuscript received January 25, 1995; revision accepted for publication June 1, 1995.) 